\title{
Probing drifting and nulling mechanisms through their interaction in PSR B0809+74
}

\author{
A. G. J. van Leeuwen ${ }^{1}$, B. W. Stappers ${ }^{2,3}$, R. Ramachandran ${ }^{2,3}$, and J. M. Rankin ${ }^{3,4}$ \\ 1 Astronomical Institute, Utrecht University, PO Box 80000, 3508 TA Utrecht, The Netherlands \\ 2 Stichting ASTRON, PO Box 2, 7990 AA Dwingeloo, The Netherlands \\ 3 Astronomical Institute "Anton Pannekoek", Kruislaan 403, 1098 SJ Amsterdam, The Netherlands \\ ${ }^{4}$ Physics Department, University of Vermont, Burlington, VT 05405, USA
}

Received 1 July 2002 / Accepted 5 November 2002

\begin{abstract}
Both nulling and subpulse drifting are poorly understood phenomena. We probe their mechanisms by investigating how they interact in PSR B0809+74. We find that the subpulse drift is not aliased but directly reflects the actual motion of the subbeams. The carousel-rotation time must then be over $200 \mathrm{~s}$, which is much longer than theoretically predicted.

The drift pattern after nulls differs from the normal one, and using the absence of aliasing we determine the underlying changes in the subbeam-carousel geometry. We show that after nulls, the subbeam carousel is smaller, suggesting that we look deeper in the pulsar magnetosphere than we do normally. The many striking similarities with emission at higher frequencies, thought to be emitted lower too, confirm this. The emission-height change as well as the striking increase in carousel-rotation time can be explained by a post-null decrease in the polar gap height. This offers a glimpse of the circumstances needed to make the pulsar turn off so dramatically.
\end{abstract}

Key words. stars: neutron - pulsars: general - pulsars: individual: PSR B0809+74 - magnetic fields radiation mechanisms: non-thermal

\section{Introduction}

In pulsars, the emission in individual pulses generally consists of one or more peaks ("subpulses"), that are much narrower than the average profile and the brightness, width, position and number of these subpulses often vary from pulse to pulse.

In contrast, the subpulses in PSR B0809+74 have remarkably steady widths and heights and form a regular pattern (see Fig. 1a). They appear to drift through the pulse window at a rate of $-0.09 P_{2} / P_{1}$, where $P_{2}$ is the average longitudinal separation of two subpulses within one rotational period $P_{1}$, which is $1.29 \mathrm{~s}$. Figure 1a also shows how the pulsar occasionally stops emitting, during a so-called null.

In this paper, we will interpret the drifting subpulse phenomenon in the rotating carousel model (Ruderman \& Sutherland 1975). In this model, the pulsar emission originates in discrete locations ("subbeams") positioned on a circle around the magnetic pole. The circle rotates as a whole, similar to a carousel, and is grazed by our line of sight. In between successive pulses, the carousel rotation moves the subbeams through this sight line, causing the subpulses to drift.

Generally, the average profiles of different pulsars evolve with frequency in a similar manner: the profile is narrow at high

Send offprint requests to: Joeri van Leeuwen,

e-mail: jleeuwen@astro.uu.nl

More information: http://www. astro.uu.nl/ jleeuwen/ frequencies and broadens towards lower frequencies, occasionally splitting into a two-peaked profile (Kuzmin et al. 1998). This is usually interpreted in terms of "radius to frequency mapping", where the high frequencies are emitted low in the pulsar magnetosphere. Lower frequencies originate higher, and as the dipolar magnetic field diverges the emission region grows, causing the average profile to widen.

The profile evolution seen in PSR B0809+74 is different. The movement of the trailing edge broadens the profile as expected, but the leading edge does the opposite. The profile as a whole decreases in width as we go to lower frequencies until about $400 \mathrm{MHz}$. Towards even lower frequencies the profile then broadens somewhat (Davies et al. 1984; Kuzmin et al. 1998). Our own recent observations of PSR B0809+74, simultaneously at 382, 1380 and $4880 \mathrm{MHz}$, confirm these results (Rankin et al. 2002). Why the leading part of the expected profile at $400 \mathrm{MHz}$ is absent is not clear. While Bartel et al. (1981) suggest cyclotron absorption, Davies et al. (1984) conclude that the phenomenon is caused by a non-dipolar field configuration. We will refer to this non-standard profile evolution as "absorption", but none of the arguments we present in this paper depends on the exact mechanism involved.

In a recent paper (van Leeuwen et al. 2002, henceforth Paper I) we investigated the behaviour of the subpulse drift in general, with special attention to the effect of nulls. We found that after nulls the driftrate is less, the subpulses are wider but 


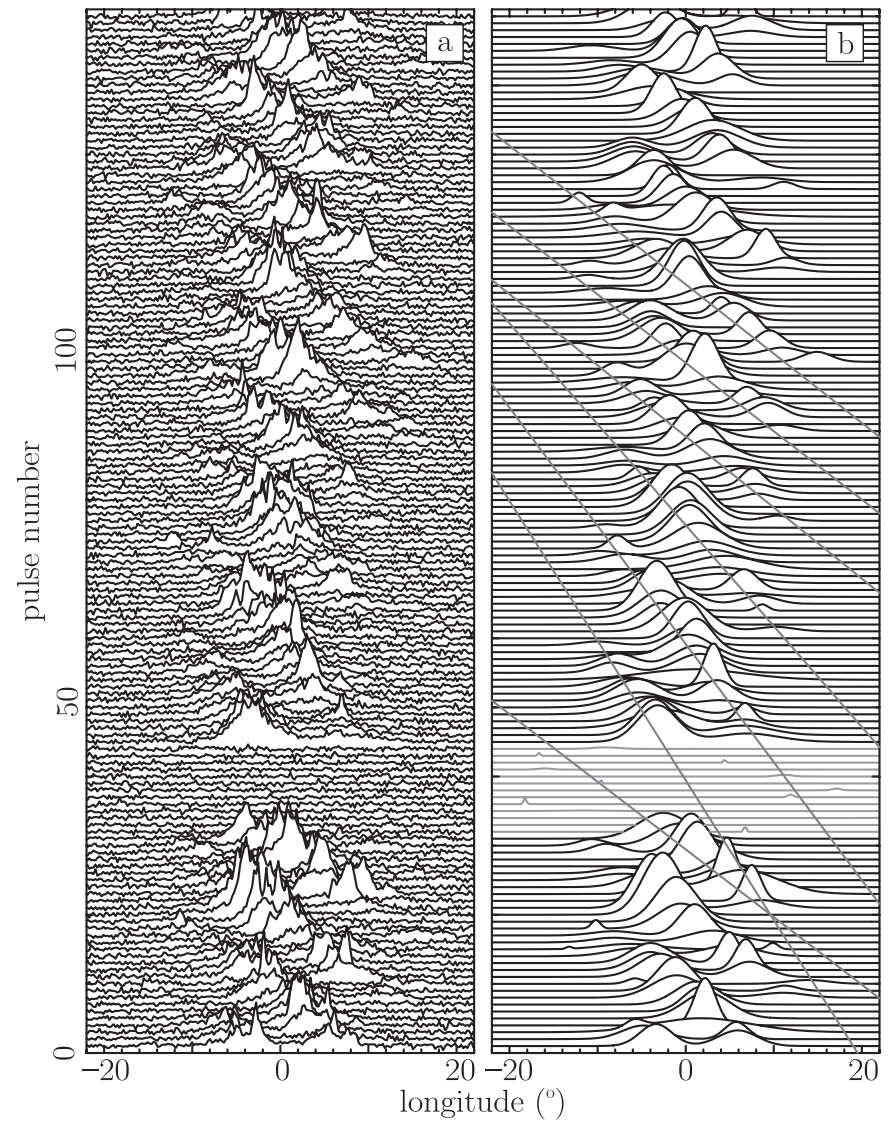

Fig. 1. Observed and fitted pulse sequences. A window on the pulsar emission is shown for 150 pulses. One pulse period is $360^{\circ}$. The centre of the Gaussian that fits the pulse profile best is at $0^{\circ}$. a) The observed pulse sequence, with a null after pulse 30 . b) The Gaussian curves that fitted the subpulses best. Nulls are shown in lightest gray, driftbands fitted to the subpulse pattern are medium gray.

more closely spaced, and the average pulse profile moves towards earlier arrival. Occasionally this post-null drift pattern remains stable for more than $150 \mathrm{~s}$.

For a more complete introduction to previous work on PSR B0809+74, as well as for information on the observational parameters and the reduction methods used, we refer the reader to Paper I. In this paper we will investigate the processes that underly the post-null pattern changes. We will quantify some of the timescales associated with the rotating carousel model and map the post-null changes in the drift pattern onto the emission region.

One of the interesting timescales is the time it takes one subbeam to complete a rotation around the magnetic pole. This carousel-rotation time is predicted to be of the order of several seconds in the Ruderman \& Sutherland model. Only recently a carousel-rotation time was first measured: Deshpande \& Rankin (1999) find a periodicity associated with a 41-s carousel-rotation time for PSR B0943+10.

The second goal is to determine the changes in the emission region that underly the different drift pattern we see after nulls. Mapping this emission region could increase our insight into what physically happens around nulls.
Achieving either goal requires solving the so-called aliasing problem: as the subpulses are indistinguishable and as we observe their positions only once every pulse period, we cannot determine their actual speed.

\section{Solving the aliasing problem}

The main obstacles in the aliasing problem are the under sampling of the subpulse motion and our inability to distinguish between subpulses. The pulsar rotation only permits an observation of the subpulse positions once every pulse period. Following them through subsequent pulses might still have led to a determination of their real speed, but unfortunately the subpulses are so much alike that a specific subpulse in one pulse cannot be identified in the next, making it impossible to learn its real speed.

In Fig. 2 we show a simulation of subpulse drifting, where we have marked all subpulses formed by a particular subbeam with a darker colour. We use these simulations to discuss how the driftrate, which is the observable motion of the subpulses through subsequent pulses, is related to the subbeam speed, which cannot be determined directly. In Fig. 2a the speed of the subbeams is low $\left(-0.09 P_{2} / P_{1}\right)$ and identical to the driftrate. In Fig. $2 \mathrm{~b}$ the subbeam speed is higher $\left(0.91 P_{2} / P_{1}\right)$, but the driftrate is identical to the one seen in Fig. 2a. When the differences between subpulses formed by various subbeams are smaller than the fluctuations in subpulses from one single subbeam, these two patterns cannot be distinguished from one another. In that case the subpulses within one driftband, which seem to be formed by one subbeam, can actually be formed by a different subbeam each pulse period ("aliasing").

To solve the aliasing problem for PSR B0809+74 we follow driftrate changes after nulls to determine the subbeam speed. Nulls last between 1 and 15 pulse periods, and in Paper I we have shown that for each null the positions of the subpulses before and after the null are identical if we correct for the shift of the pulse profile. So, as there is no apparent shift in subpulse position, either the subbeams have not moved at all, or their movement caused the new subpulses to appear exactly at the positions of the old ones.

As the lengths of the nulls are drawn from a continuous sample it is highly unlikely that the subbeam displacement is always an exact multiple of the subpulse separation: only a total stop of the subbeam carousel can explain why the subpulse positions are always unchanged over the null. At some point after the null, however, the subbeams have accelerated, and the drift pattern has returned to normal. In Fig. 1 we see how, after a null, the driftrate increases to its normal value in about 50 pulses.

There are two scenarios for this subbeam acceleration. The first we will call gradual speedup. Here the changes in the subbeam speed occur on timescales larger than $P_{1}$. The second we will call instantaneous, as the entire acceleration happens within $1 P_{1}$, effectively out of sight.

In Fig. 3 we show four simulated pulse sequences with different speedup parameters. In all cases, we simulate a drift pattern like that of PSR B0809+74. During a null, from pulses 30 to 45 , there is no subbeam displacement. Immediately after 


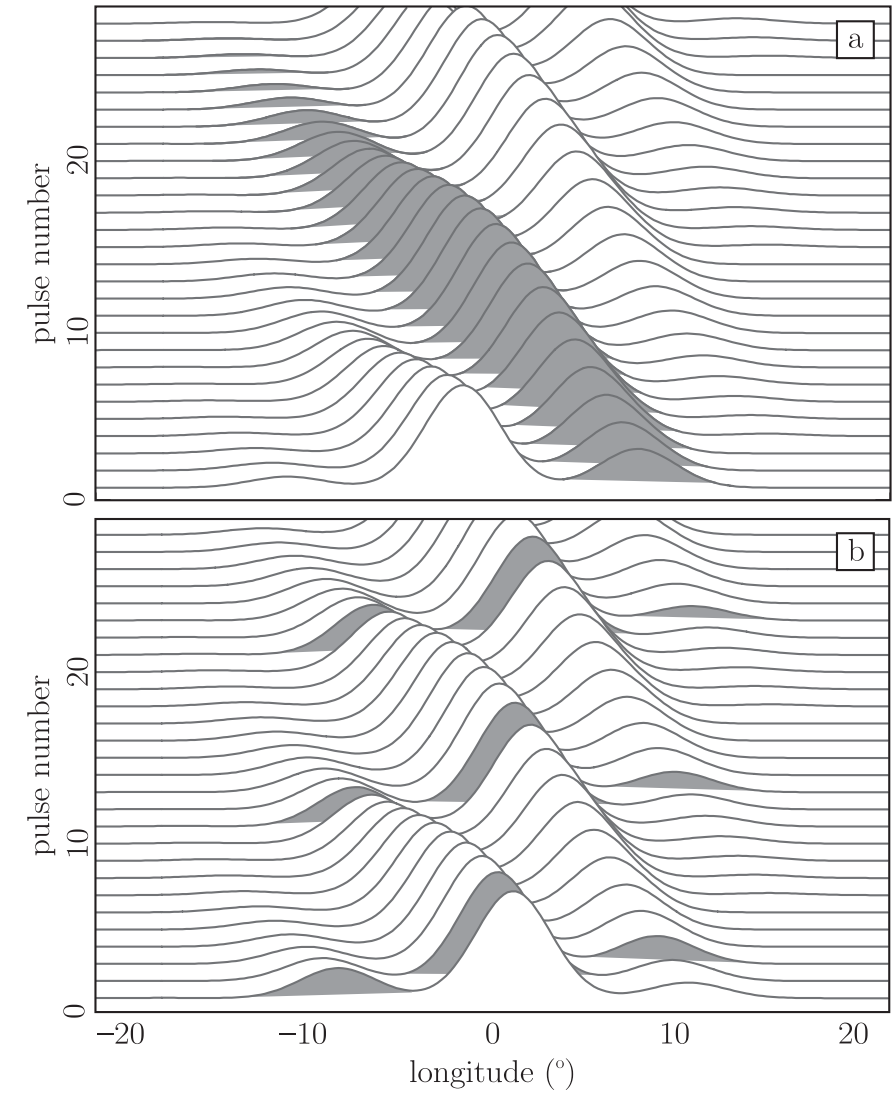

Fig. 2. Different alias orders illustrated. We show two series of stacked simulated drifting subpulses. We have marked the subpulses formed by one particular subbeam with a darker colour. a) At a low subbeam speed, a single subbeam traces an entire driftband by itself. The driftrate is identical to the subbeam speed: alias order 0 . b) At alias order -1 , the subbeam speed is higher than the driftrate and in opposite direction.

the null the subbeams build up speed, and each pulse period we translate the subbeam displacement to a change in subpulse position. Although the final driftrate is the same for all scenarios $\left(-0.09 P_{2} / P_{1}\right)$, the subbeam speeds differ considerably. The bottom four graphs show these speeds for each scenario. In the top four diagrams we have marked the subpulses from one subbeam with a darker colour for clarification.

Let us look at the case of gradual speedup to alias order 0 , where the subbeam speed is the same as the driftrate (Fig. 3a). In this case the driftrate will gradually increase and form a regular driftband pattern, much like the pattern found in the observations.

Next, we investigate a subbeam acceleration to a slightly higher speed. At $0.91 P_{2} / P_{1}$, Fig. $3 \mathrm{~b}$ shows alias mode -1 , which is the simplest configuration in which the subbeams move opposite to the subpulse drift. Right after the null the drift consequently commences in this opposite direction. When the subbeam speed nears the first aliasing boundary $0.5 P_{2} / P_{1}$, the subpulses seem to move erratically through the window. After the subbeam speed passes the first alias boundary, the subpulse drift resumes its normal direction, and as the subbeam speed approaches $0.91 P_{2} / P_{1}$, the drift pattern returns to normal.
Also in the "alias order 1" scenario (Fig. 3c), which is the simplest aliased mode in which the subbeams move in the same direction as the subpulses, the subpulses wander when the subbeam speed nears an alias boundary. Such a disturbance of the drift pattern turns out to be present in all simulations of nonzero alias orders. Because the observed pulse sequences always show smooth, non-wandering driftbands (like in Fig. 1a), we conclude that the subbeams cannot accelerate gradually to a high speed.

With instantaneous acceleration the subbeam speed switches suddenly. Most likely it will do so when the pulsar beam faces away from us. After we see pulse number $n$, the subbeams will move slowly for a certain time, quickly speed up, and then move fast until we see the subpulses of pulse $n+1$ appear. The actual speedup can occur any moment between seeing pulses $n$ and $n+1$. This means that the displacement of the subbeams can vary from very little (speedup just before pulse $n+1$ ) to a lot (speedup right after pulse $n$ ). The accompanying changes in subpulse position will then be evenly distributed between 0 and $P_{2}$; the subpulse positions after the speedup will not be related to those before.

If the subbeams accelerate instantaneously at the end of a null, before the first pulse can be observed, the subpulse phases are not preserved over the null. As this is opposite to what is observed, this possibility is ruled out.

If the subbeam acceleration occurs a few pulses after a null, we expect that the change in driftrate will nearly always be accompanied by a sudden change in the longitude of the driftband, as illustrated in Fig. 3d for a final subbeam speed of $-1.09 P_{2} / P_{1}$. The absence of a significant number of such sudden shifts in the data indicates that the subbeam carousel of PSR B0809+74 does not speed up instantaneously to a high speed.

As only the gradual speedup to alias order 0 can explain the observed drift patterns, the drift seen in the subpulses of PSR B0809+74 directly reflects the movement of the subbeams, without any aliasing.

\section{Discussion}

\subsection{Alias order of other pulsars}

The subbeam speedup in PSR B0809+74 follows the simplest scenario possible: it is gradual, and the subpulse drift is not aliased. In other pulsars this might not be the case, and for those we predict drift-direction reversals or jumps in driftband longitude during the subpulse-drift speedup phase after nulls.

\subsection{Subbeam-carousel rotation time}

Having resolved the aliasing of the subpulses' representation of the subbeams, we know that the subbeams move at $-0.09 P_{2} / P_{1}$. Such a low rotation rate means it will take each subbeam $11 P_{1}$ to move to the current position of its neighbour. The following estimate of the number of subbeams then leads directly to the carousel-rotation time.

Towards the edges of the profile the sight line and the carousel move away from each other, and a subbeam will cease 

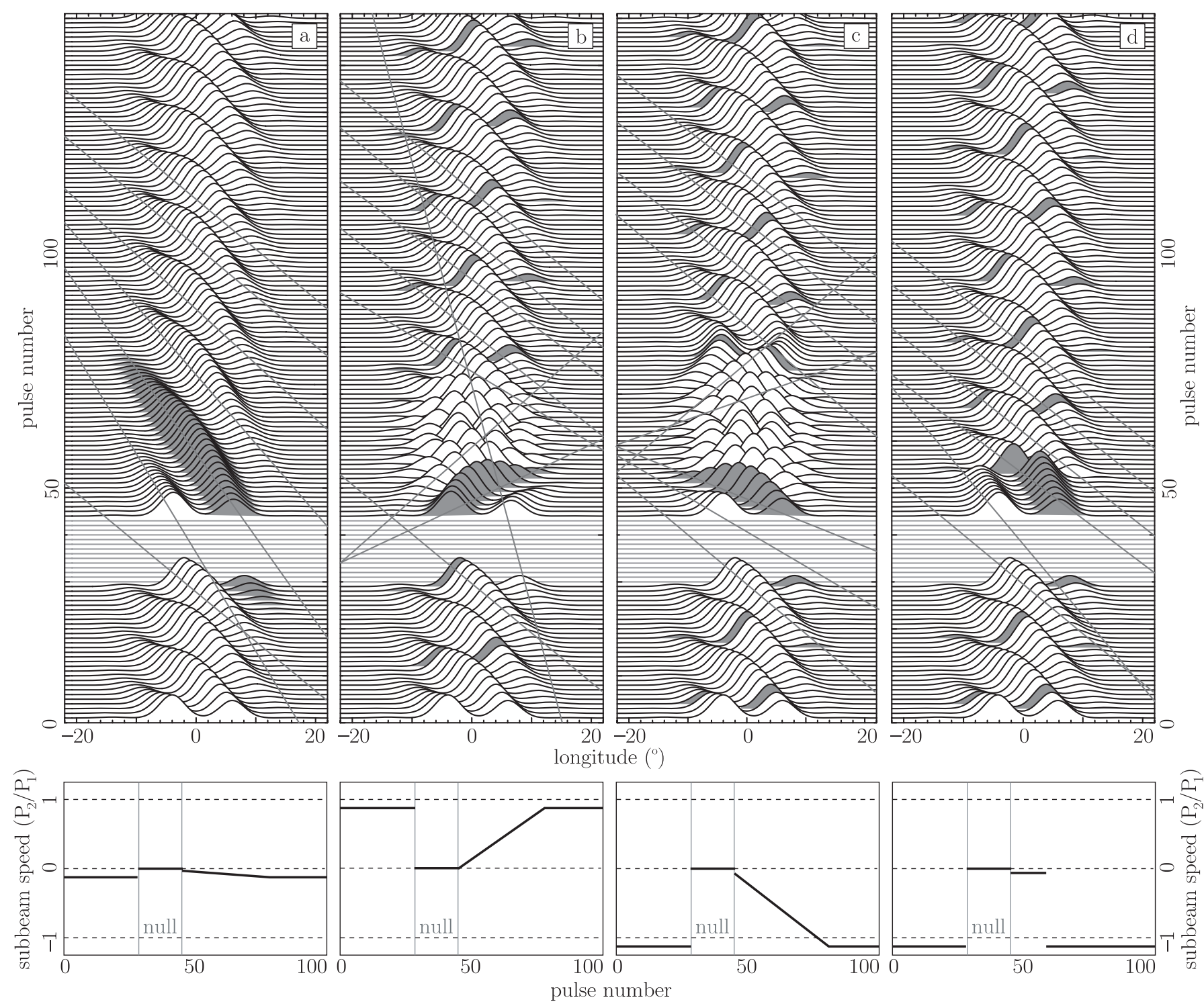

Fig. 3. Simulated scenarios for subbeam speedup. The top panels show the resulting drift patterns. Nulls are shown in lightest gray, driftbands fitted to the subpulse pattern are overdrawn in medium gray. We have marked the subpulses formed by one particular subbeam with a darker colour. The bottom panels show the subbeam speed versus the pulse number. a) Gradual acceleration to a subbeam speed equal to the driftrate (alias order 0). b) Gradual acceleration to a subbeam speed larger than the driftrate and in opposite direction (alias order -1) c) Gradual acceleration to a subbeam speed larger than the driftrate and in the same direction (alias order 1) d) Instantaneous acceleration, again to alias order 1 .

to be visible when the sight line no longer crosses it. When the subbeams are small, or widely spaced compared to the curvature of the carousel, the sight line will cross only few. In that case there will not be many subpulses visible in one pulse. If, however, the subbeams are large and closely spaced compared to the carousel curvature, the sight line passes over more subbeams in one traverse, leading to many subpulses per pulse.

In most of the pulses of PSR B0809+74 we observe two subpulses, occasionally we discern three. By combining this with the ratio of the subpulse width and separation we find there must be more than 15 subbeams on the carousel. As one subbeam reaches the position of its neighbour in $11 P_{1}$, a $15-$ subbeam carousel rotates in over $200 \mathrm{~s}$.

Persistent differences in the properties of individual subbeams should introduce a long term periodicity in the pulse sequences, at the carousel-rotation frequency. Thus far, no such periodicity has been found, which is not surprising as the periodicity can only be measured if the lifetime of the subbeam characteristics is longer than the carousel-rotation time.

In PSR B0943+10, the only pulsar for which we know how the subbeams vary (Deshpande \& Rankin 1999, 2001), the associated timescales are in the order of $100 \mathrm{~s}$. If we assume their lifetimes are comparably long in PSR B0809+74, the subbeams will have lost most of their recognisable traits once they return into view after one carousel rotation. Having regular pulse sequences that contain several tens of rotation times might still show some periodicity at the carousel-rotation frequency. Unfortunately nulls have a destructive influence on the driftband pattern and possibly on the subpulse characteristics. This has thus far made it impossible to observe bright 


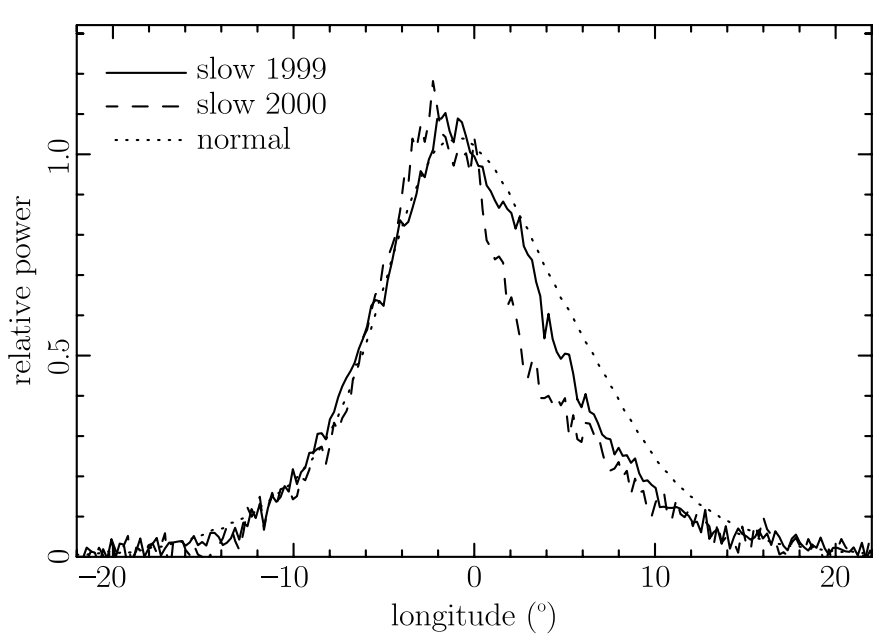

Fig. 4. Pulse profiles for the normal mode and the two longest slow drifting sequences after nulls described in Paper I. The broadening of the subpulses causes the slow-drifting profile to outshine the normal one. Here all three profiles have been scaled to the same height to show the change in the pulse location and shape.

sequences longer than 3 times our lower limit on the rotation time of $200 \mathrm{~s}$.

After the prediction that the rotation time in most pulsars would be on the order of several seconds (Ruderman \& Sutherland 1975), the observation that it is $41 \mathrm{~s}$ in PSR B0943+10 was somewhat surprising. The suggested dependence of the carousel-rotation time on the magnetic field strength and the pulse period predicts that the rotation time in PSR B0809+74 should be roughly 4 times smaller. The circulation time we observe, several hundreds of seconds, shows that the theoretical predictions are incorrect in absolute numbers as well as in the scaling relations they propose.

\subsection{Emission region geometry}

In Paper I we found many interesting changes in the driftband pattern after nulls. The subpulses reappear 5-10\% broader and $15 \%$ closer together, which results in a brighter average profile. The individual subpulses move $1.5^{\circ}$ towards earlier arrival, as does their envelope, the average profile, with $1.1^{\circ}$ (Fig. 4). The subpulses drift $50 \%$ more slowly than usual. Occasionally, this slow drifting pattern remained stable for over 100 pulses.

Knowing, as we do now, that there is no aliasing involved, we can identify single subpulses with individual subbeams. This implies that we can interpret the drift pattern changes in terms of the subbeam-carousel geometry (Fig. 5). We will do so for each of the altered characteristics.

The first thing we note is that, relative to the shift of the average profile, the positions of the subpulses are unchanged over nulls. This means that the subbeam carousel has not rotated during the null.

After nulls, the subpulses are positioned about $15 \%$ closer together. In principle, this could be the result from a change in subbeam speed. At a certain moment, a particular subbeam will be pointing towards the observer. It takes some time before the pulsar has rotated the next subbeam into the observer's view, and during this time the subbeams themselves have moved, too. This motion translates directly to a change in the longitudinal subpulse separation. Yet, as we have shown that subbeam speed is low, this effect is negligible.

A more significant change in $P_{2}$ could be caused by an increase in the number of subbeams on a carousel of unchanged size. The other option is that a decrease in the carousel radius moves the subbeams closer together.

In the first scenario the number of subbeams would have to change during the null, causing the subbeam placement to change considerably. This would lead to subpulse-position jumps over the null. In that case, we would not expect the phases of the subpulses to be as unchanged over the nulls as is observed. Secondly, some time after the null the new configuration would have to return to normal. This means subbeams would have to appear or disappear. We see no evidence of this in any of the 200 nulls we observed. The change in the subbeam separation $P_{2}$ can therefore not be due to a changed number of subbeams.

In the second scenario the carousel radius decreases by $15 \%$, but the number of subbeams remains the same. As the subbeams now share a reduced circumference, their separation also decreases.

The contraction of a carousel causes all subpulses to move towards the longitude of the magnetic axis. If we combine this contraction with the aforementioned absorption, we can immediately explain the observed shift to earlier arrival of the subpulses and their envelope, the average profile: with only the trailing part unabsorbed, all visible subpulses move towards earlier arrival (Figs. 4 and 5).

In general, the subpulses farthest from the longitude of the magnetic axis will move most, while those at it, if visible, will remain at their original positions. Enlargement of our sample of subpulse positions can therefore indicate were the magnetic axis of PSR B0809+74 is located. This would immediately indicate how much of the pulse profile is "absorbed" and illuminate the thus far much debated alignment of the pulse profiles at different frequencies.

Normally, the edges of a pulse profile indicate where the overlap of the sight line and the subbeam carousel begins and ends. The contraction of a carousel will then move both edges inward. Yet when the first part of the profile is absorbed, the leading edge reflects the end of the absorption. If this edge is near the middle of the sight-line traverse over the carousel, it will not be affected by a reduction of the carousel size. In that case we will see a change in the position of the trailing edge but not in the leading one, which is exactly what we observe in PSR B0809+74 (Fig. 4).

The wider subpulses we see after a null translate directly to wider subbeams. We do note that a change in carousel radius leads to a new sight-line path, which may have an impact as well.

The significant chance that a null starts or ends within the pulse window of the pulses that surround it, would on average make these pulses dimmer than normal ones (Lyne \& Ashworth 1983). Quite unexpectedly however, the pulses after nulls were found to be brighter than average. This is easily explained in our model: the change in the carousel geometry causes the 

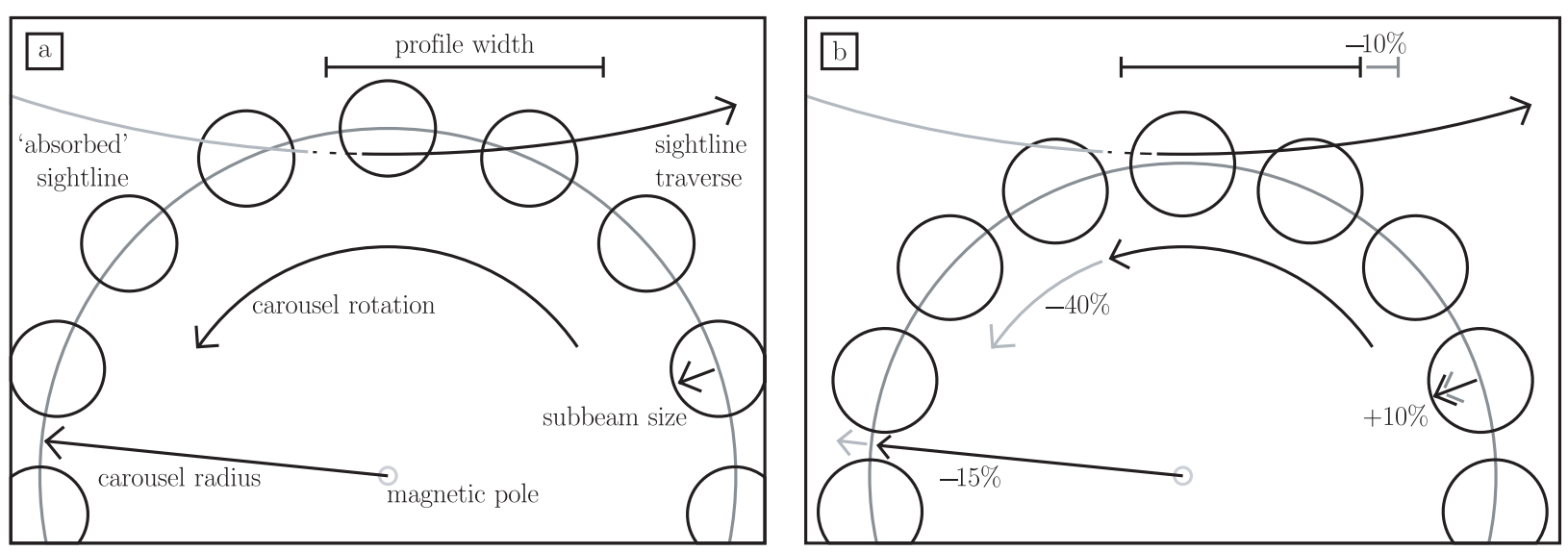

Fig. 5. Subbeam carousel geometry in the normal and the post-null configuration. a) The normal configuration, with a sight line that is partially obscured by the absorption. b) After nulls the carousel is smaller. As their number is unchanged, the subpulses have moved closer together. On the non-absorbed, trailing edge, the pulse window moves inward. The leading edge of the profile is located where the absorption ends and does not move. The subpulses are wider and revolve less fast.

subpulses to broaden and become more closely spaced, while their peak brightness remains the same. This leads to the postnull pulse-intensity increase that is observed.

\subsection{Post-null polar gap height}

If we follow the radius-to-frequency-mapping argument discussed in the introduction, then the reduction of the carousel size implies that the emission after a null comes from lower in the pulsar magnetosphere than it does normally. The reduction of the subbeam separation supports this idea, but the broadening of the subpulses seems inconsistent.

At this stage a comparison with the pulse profiles and drift patterns observed at higher frequencies seems promising. Emission at higher frequencies is also supposed to originate lower in the pulsar magnetosphere, so similar effects may point at one cause: a decrease in emission height.

Much like the post-null profile, the average profile at higher frequencies moves towards earlier arrival (Rankin et al. 2002). Comparing drift patterns, we see that at higher frequencies the driftrate decreases and that the subpulses broaden and move closer to one another (Davies et al. 1984), strikingly similar to the behaviour we find after nulls.

Only one anomaly remains: when comparing drift patterns at different frequencies, the changes in driftrate and subpulse separation $P_{2}$ always counterbalance, leaving the carousel rotation time $\hat{P}_{3}$ unchanged. This means that the subbeams we observe at a certain frequency are cuts through rods of emission, intersections at the emission height associated with that frequency. The rods rotate rigidly but diverge with increasing height. At each height $\hat{P}_{3}$ is the same, while the speed and separation of the subbeams do change.

In contrast to this normal invariance of $\hat{P}_{3}$, we see it change considerably after nulls, an increase that cannot be explained by a change in viewing depth. We therefore expect that the disturbance that caused the change in this depth, can also account for the $50 \%$ increase in $\hat{P}_{3}$.
Usually, both the subbeam separation $P_{2}$ and the emission height are assumed to scale with the polar gap height $h$ (Ruderman \& Sutherland 1975; Melikidze et al. 2000). The $15 \%$ decrease in $P_{2}$ would thus be due to a identical fractional decrease in the gap height, which should also cause an equal decrease in emission height.

If this change in gap height could also account for the observed increase of the carousel rotation time $\hat{P}_{3}$, all post-null drift-pattern changes can be attributed to one single cause.

$\hat{P}_{3}$ is thought to scale as $h^{-2}$. For the inferred $15 \%$ gap height decrease this predicts a $40 \%$ increase in $\hat{P}_{3}$, nicely similar to the $50 \%$ we find.

With one single cause we can therefore explain both the puzzling well-known phenomena (the driftrate decrease after nulls, the bright first active pulse) and the newly discovered subtle ones (the change in the position of the average profile, the decrease of the subpulse separation and the subpulse-width increase). This post-null decrease in gap height offers a glimpse of the circumstances needed to make the pulsar turn off so dramatically.

\section{Conclusions}

We have shown that the drift of the subpulses directly reflects the actual motion of the subbeams, without any aliasing.

In other pulsars with drifting subpulses this may be different: for those we predict drift-direction reversals or longitude jumps in the post-null drift pattern.

We find that the carousel-rotation time for PSR B0809+74 must be long, probably over $200 \mathrm{~s}$. The expected lifetime of the subbeam characteristics is less, which explains why thus far no periodicity from the carousel rotation could be found in the pulse sequence.

The rotation time we find is larger than theoretically predicted, not only in absolute numbers but also after extrapolating the rotation time found in PSR B0943+10. Both the magnitude and the scaling relations that link the carousel-rotation time to the magnetic field and period of the pulsar are therefore incorrect. 
When the emission restarts after a null the drift pattern is different, and having determined the alias mode, we identify the underlying changes in the geometry of the subbeam carousel. A combination of a decrease in carousel size and "absorption" already explains many of the changes seen in the post-null drift pattern.

The resemblance between the drift pattern after nulls and that seen at higher frequencies, thought to originate at a lower height, is striking. Assuming that similar effects have identical causes leads us to conclude that after nulls we look deeper in the pulsar magnetosphere, too.

Both this decrease in viewing depth and the striking increase in the carousel rotation time can be quantitatively explained by a post-null decrease in gap height.

Acknowledgements. We thank Marco Kouwenhoven for his help with the observations and Frank Verbunt, Russell Edwards and Patrick Weltevrede for constructive discussions.

\section{References}

Bartel, N., Kardashev, N. S., Kuzmin, A. D., et al. 1981, A\&A, 93, 85 Davies, J. G., Lyne, A. G., Smith, F. G., et al. 1984, MNRAS, 211, 57 Deshpande, A. A., \& Rankin, J. M. 1999, ApJ, 524, 1008

Deshpande, A. A., \& Rankin, J. M. 2001, MNRAS, 322, 438

Kuzmin, A. D., Izvekova, V. A., Shitov, Y. P., et al. 1998, A\&AS, 127, 255

van Leeuwen, A. G. J., Kouwenhoven, M. L. A., Ramachandran, R., Rankin, J. M., \& Stappers, B. W. 2002, A\&A, 387, 169

Lyne, A. G., \& Ashworth, M. 1983, MNRAS, 204, 519

Melikidze, G. I., Gil, J. A., \& Pataraya, A. D. 2000, AJ, 544, 1081

Ramachandran, R., Rankin, J. M., Stappers, B. W., Kouwenhoven, M. L. A., \& van Leeuwen, A. G. J. 2002, A\&A, 381, 993

Rankin, J. M., Ramachandran, R., van Leeuwen, A. G. J., Suleymanova, S. A., \& Deshpande, A. A. 2002, A\&A, in preparation

Ruderman, M. A., \& Sutherland, P. G. 1975, ApJ, 196, 51 